\title{
Optimization of Voltage Doublers for Energy Harvesting Applications
}

\author{
Nina M. Roscoe and Martin D. Judd
}

\begin{abstract}
Energy harvesting is increasingly enabling the expansion of wireless sensor networks in challenging applications by replacing batteries in low power sensors. Many forms of energy harvester suffer from low output voltage which can be partially compensated for by the use of a Cockcroft-Walton voltage doubler ahead of a dc-dc converter. Impedance matching of energy harvesters is critical to achieving high output power per unit volume. This paper explores optimum impedance match for an energy harvester with a voltage doubler and dc-dc converter. Formulae are derived, and experimentally confirmed, which calculate optimum impedance match between the harvester and a load, and calculate voltage at the input to the dcdc converter for a given wireless sensor power consumption. Further, the formula for optimum impedance match is validated against independently published results.
\end{abstract}

Index Terms - Energy harvesting, voltage doubler, CockcroftWalton, magnetic field, inductive harvesting.

\section{INTRODUCTION}

$\mathrm{E}$ nergy Harvesting techniques adapted for a wide range of applications, amongst which wireless sensors dominate, are rapidly increasing in number and improving in performance as technologies mature. Energy harvesting is the enabling technology for the application of wireless sensors to many industrial applications where hard-wired power is not available, and batteries cannot be changed due to a combination of inaccessibility, excessively numerous batteries and risk to personal safety [1]. The most common energy sources for harvesters include wind [2,3] solar [4,5] piezoelectric vibration [6], electromagnetic vibration $[7,8]$ and Magnetic Shape Memory (MSM) vibration [9] while thermal [10], current transformer (CT) inductive electromagnetic $[11,12]$, free-standing inductive $[1,13]$ and capacitive electromagnetic $[15,16]$ provide energy in spaces where wind, solar and vibration energy are not available in sufficient quantity.

A number of energy harvesting techniques suffer from low

Manuscript received June 12, 2013. This work was supported and funded by National Grid ref: 227819/FFR.

N.M. Roscoe is with the High Voltage Technology Research Group, University of Strathclyde, Glasgow (Corresponding author, Tel: +44 141548 2120; fax: +44 141552 5398; e-mail: nina.roscoe@strath.ac.uk).

M.D. Judd is with the High Voltage Technology Research Group, University of Strathclyde, Glasgow (e-mail: m.judd@ strath.ac.uk).

Copyright (c) 2012 IEEE. Personal use of this material is permitted. However, permission to use this material for any other purposes must be obtained from the IEEE by sending a request to pubs-permissions@ieee.org. output voltage due to factors such as low source impedance or low available energy $[8,11,14]$. Output voltage must therefore be boosted before it can be used to power electronics. One solution is to boost voltage with a Cockcroft-Walton voltage multiplier $[1,8,11,12]$, which provides full-wave rectification but with lower diode conduction losses than a conventional bridge rectifier.

Cockcroft-Walton voltage multipliers employ the charge pump principle to accumulate a dc voltage on capacitors from an ac voltage source, using low voltage Schottky diodes as switches. When terminated with a very high impedance load, each stage of the multiplier develops a dc output voltage roughly equal to double the peak input voltage, and is therefore referred to as a doubler. The conversion loss of the doubler stage is determined by the ratio of input ac voltage to Schottky diode forward voltage drop [17]. When input voltage is very small, many stages of multiplication will be required to boost harvester output voltage to a level compatible with a dcdc upconverter. Efficiency degrades considerably as the number of stages increases due to the increased number of diodes. Therefore, if more than one stage of doubling is required to achieve sufficient input voltage to operate a dc-dc upconverter, then the Cockcroft-Walton solution is usually too inefficient. An alternative solution in such cases is to use an ac-dc boost converter [14] or an active rectifier for which the dual rail supply is provided by a subsidiary coil with dickson charge-pump and half-wave rectifier [18]. However, when the available voltage is almost high enough for commercial dc-dc converters, the Cockcroft-Walton doubler (from now on referred to as doubler for simplicity) provides a simple, easily implemented solution. Using a Cockcroft-Walton opens up the possibility of using a very high efficiency dc-dc converter, such as that described in [12]. For applications where peak harvester output voltage is between 0.5 and $1 \mathrm{~V}$, the voltage doubler can be an efficient and robust solution when combined with a dc-dc converter.

To maximize available output power from any energy harvester it is important to terminate the harvester with a matched impedance. Connecting a voltage doubler to the output of the harvester modifies the impedance presented by the harvester to the load. In order to optimize the performance of a harvester which is using a voltage doubler, it is therefore necessary to understand how the voltage doubler affects the impedance match between the load and the harvester. In this paper operation of the voltage doubler is analyzed to determine the optimum load impedance that should be applied 
to a harvester which uses a voltage doubler. An expression for the power delivered to the load is derived, taking leakage into account. The resulting formula yields a value for the optimum load resistance and the optimum value of series compensation capacitance as a function of the harvester source impedance. The analysis is extended to deduce a simple formula for the steady-state voltage the doubler and dc-dc converter.

\section{CIRCUIT ANALYSIS}

\section{A. Defining the circuit}

The analysis presented is based on a free-standing inductive harvester [1] that powers a wireless condition monitoring sensor using energy extracted from the ambient powerfrequency magnetic fields in electrical substations. The impedance of the coil used in the harvester can be approximated by an inductance $L_{s}$ in series with a resistance $R_{s}$. When analyzed at the frequency of operation $f$, where $\omega=2 \pi f$, this impedance can be expressed as

$$
R_{s}+j \omega L_{s}
$$

Since the impedance of any harvester can be expressed at the frequency of operation in terms of real and imaginary components, this analysis is valid for a wide range of harvesters using doublers.

An inductive harvester using a single stage doubler is shown in Fig. 1.

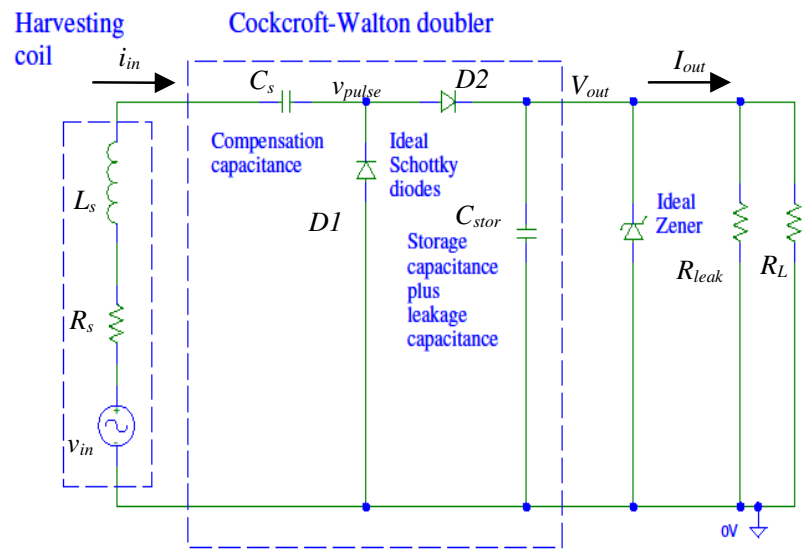

Fig. 1 Inductive harvester connected to load $R_{L}$ through a doubler.

The harvesting coil is represented by an ideal ac voltage source, $v_{i n}=V_{i n} \sin (\omega t-\theta)$, in series with the coil impedance given by (1). $v_{i n}$ is the open circuit voltage that would appear across the coil when placed in the same magnetic field, $\omega$ is the dominant frequency in the magnetic field from which the coil is harvesting and $\theta$ is the phase shift between the magnetic field and the fundamental harmonic of the diode switching. The capacitor $C_{s}$ in series with the harvesting coil has two roles: it compensates the self inductance of the coil [1] and is also the first capacitor in the doubler circuit. $C_{\text {stor }}$ is a capacitor connected across the output of the doubler to provide energy storage for periods of low magnetic flux density while also acting as the smoothing capacitor for the doubler. The diodes for the doubler are Schottky diodes chosen for low conduction loss. A shunt Zener diode protects against overvoltage at the load. The harvester in Fig. 1 is delivering power to a resistive load $R_{L}$ while leakages through $D 1, D 2, C_{\text {stor }}$ and the Zener diode are represented by a resistor $R_{\text {leak }}$ in parallel with $R_{L}$. There will also be parasitic diode capacitance but this has negligible effect on circuit behavior at the low frequencies involved in the majority of harvesting applications.

\section{B. Circuit operation}

Vibration and electromagnetic energy harvesters generate an ac voltage output. The frequency content at any one instant in time is often dominated by one frequency, in which case the harvester output is approximately sinusoidal. This is always true when harvesting from the EM field, where dominant output frequency is set by the electrical supply frequency. Furthermore, vibration harvesters applied to machinery are dominated by a single frequency, although this frequency can often vary with time [19]. This sine wave output from the harvester is applied to the input of the voltage doubler. During negative half cycles D1 conducts, so that a voltage accumulates on $C_{s}$. During positive half cycles D2 conducts, transferring charge from $C_{s}$ to $C_{s t o r}$. By inspection of the circuit it can be seen that it is not possible for both of the diodes to conduct at the same time. Capacitor values are chosen such that their charging time constant is much longer than the period of the input sine wave. In order to ascertain the output impedance for the harvester and doubler, circuit analysis is performed for the equilibrium state, where power generated by the harvester equals power delivered to $R_{L}$. Forward conduction losses in the Schottky diodes are a few $\mu \mathrm{W}$, in contrast to core loss of between 10's of $\mu \mathrm{W}$ and several $\mathrm{mW}$, at the magnetic flux density considered. Diode conduction losses are therefore neglected in the analysis.

A number of useful observations can be made about the operation of the circuit of Fig. 1 when in the equilibrium state:

1. In the steady state, the charge drained from $C_{\text {stor }}$ by the load current during the period of time where $D 2$ is off must be balanced by the charge accumulated on $C_{\text {stor }}$ while $D 2$ is on. Hence the time-average current in $D 2$ must equal $I_{\text {out }}$.

2 . The increase in voltage on $C_{s}$ while $D 1$ is on must equal the decrease in voltage on $C_{s}$ while $D 2$ is on. The voltage across $C_{s}$ is then of the form $V_{c s}+v_{c s}$ where $V_{c s}$ is a dc component onto which an ac component, $v_{c s}$, is superimposed.

3. $C_{\text {stor }}$ is large enough to provide smoothing of the output voltage, so that $V_{\text {out }}$ can be treated as constant and $I_{\text {out }}$ can be regarded as a constant dc current.

4. During the time that $D 2$ conducts, $V_{\text {out }}$ is connected to the series $L C R$ network formed by $R_{s}, L_{s}$ and $C_{s}$. During the time that $D l$ conducts, $O \mathrm{~V}$ is applied to the LCR network, so that the voltage on the cathode of $D 1, v_{\text {pulse }}=0 \mathrm{~V}$. When neither diode conducts then $v_{\text {pulse }}=v_{\text {in }}$. Hence $v_{\text {pulse }}$ can be approximated by the waveform shown in Fig. 2(a).

5 . When the power available from the harvester is matched by the power delivered to the load, then either $D 1$ or $D 2$ must be conducting at all times for maximum power transfer (when forward diode drop is neglected). Under these conditions $v_{\text {pulse }}$ is of the form shown in Fig. 2(b).

6. D1 and D2 only conduct once each in each cycle, and they conduct alternately. This implies that (i) input current 
only falls to zero twice in each cycle, (ii) the period of $v_{\text {pulse }}$ is $1 / f$, and (iii) that $f$ dominates the current spectrum.

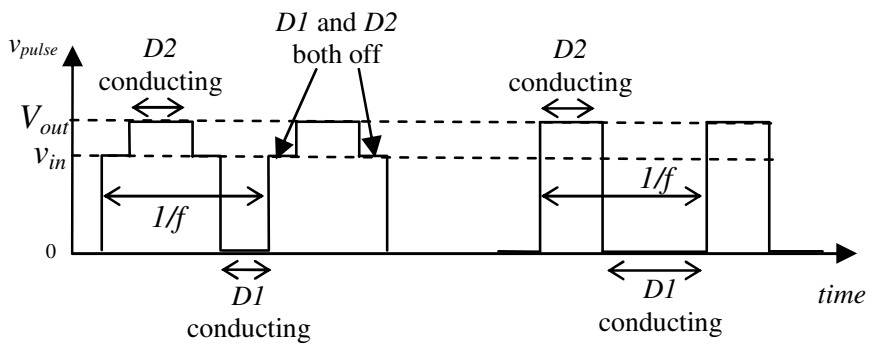

(a)

(b)

Fig. $2 v_{\text {pulse }}$ as a result of diode switching across the components $R_{s}, L_{s}$ and $C_{s}$ when (a) power delivered to load is less than available harvester power, so periods exist where both $D 1$ and $D 2$ turn off, and (b) all available harvester power is delivered to load, so diode always conducts.

\section{Waveform analysis using Fourier components}

Analysis presented here develops formulae for optimizing impedance match and predicting steady state voltage for a harvester driving a load through a doubler. For maximum power transfer in the steady state, the components $R_{s}, L_{s}$ and $C_{s}$ see a voltage across them, $v_{L C R}$, which is the difference between the input sine wave and the pulse waveform given by Fig. 2(b). Taking the pulse waveform as the phase reference, and the phase shift between the fundamental components of the pulse waveform and the input sine-wave to be $\theta$, the input sine-wave $v_{\text {in }}$ is

$$
v_{\text {in }}=V_{\text {in }} \sin (\omega t-\theta)
$$

The Fourier series expression for $v_{\text {pulse }}$ is given by

$$
v_{\text {pulse }}=\frac{V_{\text {out }} t_{d}}{T}+\frac{2 V_{\text {out }}}{\pi} \sum_{n=1}^{\infty} \frac{1}{n} \sin \frac{n \omega t_{d}}{2} \cos n \omega t
$$

where $n$ takes only odd values and $t_{d}$ is the on time for $D 2$. The total voltage across $R_{s}, L_{s}$ and $C_{s}$, is therefore

$$
\begin{aligned}
v_{L C R} & =V_{\text {in }} \sin (\omega t-\theta) \\
& -\frac{V_{\text {out }} t_{d}}{T}-\frac{2 V_{\text {out }}}{\pi} \sum_{n=1}^{\infty} \frac{1}{n} \sin \frac{n \omega t_{d}}{2} \cos n \omega t
\end{aligned}
$$

Since $R_{s}, L_{s}$ and $C_{s}$ are all passive linear components, the input current $i_{\text {in }}$ can only contain the same harmonics as $v_{\text {in }}$, so that

$$
i_{\text {in }}=\sum_{n=1}^{\infty} A_{n} \cos \left(n \omega t+\varphi_{n}\right)
$$

where $A_{n}$ are constant with time and $\varphi_{n}$ is the phase shift between the $n$th harmonic of $v_{\text {pulse }}$ and the $n$th harmonic of $i_{i n}$. A second expression for $v_{L C R}$ in terms of $i_{\text {in }}$ can then be derived, in which $V_{c s}$ is the dc voltage on $C_{s}$ at the instant D2 starts to conduct.

$$
v_{L C R}=i_{\text {in }} R_{s}+L_{s} \frac{d i_{i n}}{d t}+\frac{1}{C_{s}} \int_{t_{D 2 \_o n}}^{t} i_{i n} d t+V_{c s}
$$

Combining (5) into (6) and using trigonometric identities to expand the phase shifted terms:

$$
\begin{aligned}
v_{L C R} & =\sum_{n=1}^{\infty} A_{n}\left[\left(\frac{1}{n \omega C_{s}}-n \omega L_{s}\right) \sin \varphi_{n}+R_{s} \cos \varphi_{n}\right] \cos n \omega t \\
& +\sum_{n=1}^{\infty} A_{n}\left[\left(\frac{1}{n \omega C_{s}}-n \omega L_{s}\right) \cos \varphi_{n}-R_{s} \sin \varphi_{n}\right] \sin n \omega t \\
& +d c_{-} \text {terms }
\end{aligned}
$$

Equations (7) and (4) can now be equated to find the coefficients $A_{n}$. Two expressions are derived for $A_{l}$, given by (8a) and (8b).

$$
\begin{gathered}
A_{1}=\frac{V_{\text {in }} \sin \theta-\frac{2 V_{\text {out }}}{\pi} \sin \frac{\omega t_{d}}{2}}{\left(\frac{1}{\omega C_{s}}-\omega L_{s}\right) \sin \varphi_{1}+R_{s} \cos \varphi_{1}} \\
A_{1}=\frac{V_{\text {in }} \cos \theta}{\left(\frac{1}{\omega C_{s}}-\omega L_{s}\right) \cos \varphi_{1}-R_{s} \sin \varphi_{1}}
\end{gathered}
$$

Higher order coefficients are given by (9), and the phase of each higher order frequency component is given by (10).

$$
\begin{gathered}
A_{n}=\frac{-\frac{2 V_{\text {out }}}{\pi} \sin \frac{n \omega t_{d}}{2}}{\left(\frac{1}{\omega C_{s}}-n^{2} \omega L_{s}\right) \sin \varphi_{n}+n R_{s} \cos \varphi_{n}} \\
\tan \varphi_{n}=\frac{n\left(\frac{1}{n^{2} \omega C_{s}}-\omega L_{s}\right)}{R_{s}}
\end{gathered}
$$

Observation 6 in Section II $B$ dictates that $A_{l}>A_{n}$ for all $n \geq$ 3 , so the fundamental frequency dominates, which means that the fundamental frequency terms in both (3) and (5) must be in phase to ensure that the current in the diodes falls to zero at the same time as the diode switching takes place. Hence

$$
\varphi_{1}=0
$$

and (8a) and (8b) can be more simply expressed as

$$
\begin{gathered}
A_{1}=\frac{V_{\text {in }}}{R_{s}} \sin \theta-\frac{2 V_{\text {out }}}{\pi R_{s}} \sin \frac{\omega t_{d}}{2} \\
A_{1}=\frac{V_{\text {in }} \cos \theta}{\left(\frac{1}{\omega C_{s}}-\omega L_{s}\right)}
\end{gathered}
$$

$V_{\text {out }}$ is given by $V_{\text {out }}=\frac{P_{\text {out }}}{I_{\text {out }}}$, where $I_{\text {out }}$ is the load current (regarded as constant from observation 3 in Section II $B$ ) and $P_{\text {out }}$ is the power delivered to $R_{L}$ and $R_{\text {leak }}$. Observation 1 in Section II $B$ indicates that $I_{\text {out }}$ is equal to the average current in $D 2$. The average current in D2 is found by integrating over the positive half cycle of $i_{i n}$, and dividing by the waveform period.

$$
I_{\text {out }}=\frac{\omega}{2 \pi} \int_{-\frac{\pi}{2 \omega}}^{\frac{\pi}{2 \omega} i_{\text {in }}} d t
$$

An expression for $I_{\text {out }}$ can now be obtained by substituting (5) into (13) and performing the integration, which gives

$$
I_{\text {out }}=\frac{1}{\pi} \sum_{m=1}^{\infty} \frac{A_{n}}{n} \cos \varphi_{n}
$$

Since $A_{n}<A_{l}$ for all $n \geq 3$, then $\left(A_{n} / n\right)<<A_{1}$ for $n \geq 3$, so it is reasonable to neglect all but the fundamental in (14). Combining this with (11) leads to a simple expression for $I_{o u t}$.

$$
I_{\text {out }} \approx \frac{A_{1}}{\pi}
$$

$V_{\text {out }}$ (approximated as constant from observation 3 in Section II $B$ ) can now be expressed in terms of output power and $A_{l}$. 


$$
V_{\text {out }}=\frac{P_{\text {out }} \pi}{A_{1}}
$$

Rearranging (12b) gives the phase angle $\theta$ between $v_{\text {pulse }}$ and $v_{\text {in }}$ in terms of $A_{1}$.

$$
\theta=\cos ^{-1} \frac{A_{1}\left(\frac{1}{\omega C_{s}}-\omega L_{s}\right)}{V_{\text {in }}}
$$

Using (16) to substitute for $V_{\text {out }}$, and (17) to substitute for $\varphi$ in (12a) leads to an expression for $A_{l}$ :

$$
A_{1}=\frac{V_{\text {in }}}{R_{s}} \sin \left[\cos ^{-1} \frac{A_{1}\left(\frac{1}{\omega C_{s}}-\omega L_{s}\right)}{V_{\text {in }}}\right]-\frac{2 P_{\text {out }}}{A_{1} R_{s}} \sin \frac{\omega t_{d}}{2}
$$

The sine of the inverse cosine in (18) can be alternatively expressed using trigonometry so that

$$
A_{1}=\frac{\sqrt{V_{\text {in }}{ }^{2}-A_{1}^{2}\left(\frac{1}{\omega C_{s}}-\omega L_{s}\right)^{2}}}{R_{s}}-\frac{2 P_{\text {out }}}{A_{1} R_{s}} \sin \frac{\omega t_{d}}{2}
$$

This expression is a quadratic in $A_{l}^{2}$ whose roots are given by (20) with constants $k_{l}$ and $k_{2}$ defined by (21) and (22).

$$
\begin{gathered}
A_{1}^{2}=\frac{-k_{1} \pm \sqrt{k_{1}^{2}-16 k_{2} P_{\text {out }}{ }^{2} \sin ^{2} \frac{\omega t_{d}}{2}}}{2 k_{2}} \\
k_{1}=4 P_{\text {out }} \sin \frac{\omega t_{d}}{2} R_{s}-V_{\text {in }}{ }^{2} \\
k_{2}=R_{s}^{2}+\left(\frac{1}{\omega C_{s}}-\omega L_{s}\right)^{2}
\end{gathered}
$$

$P_{\text {out }}$ can be expressed in terms of $I_{\text {out }}$ and the parallel combination of $R_{L}$ and $R_{\text {leak }}$, in which (15) is used to substitute for $I_{\text {out }}$.

$$
P_{\text {out }}=I_{\text {out }}{ }^{2}\left(R_{L} / / R_{\text {leak }}\right)=\frac{A_{1}^{2}}{\pi^{2}}\left(R_{L} / / R_{\text {leak }}\right)
$$

A new expression for $A_{1}$ can be found by substituting for output power in (20) using (23) and rearranging to give

$$
A_{1}^{2}=\frac{-k_{1}}{k_{2}+4 \frac{\left(R_{L} / / R_{\text {leak }}\right)^{2}}{\pi^{4}} \sin ^{2} \frac{\omega t_{d}}{2}}
$$

Of the power $P_{\text {out }}$ delivered by the doubler in (23) some power is dissipated in $R_{\text {leak }}$ while the rest of the power $P_{d e l}$ is delivered to $R_{L}$.

$$
P_{\text {del }}=P_{\text {out }} \frac{\left(R_{L} / / R_{\text {leak }}\right)}{R_{L}}=\frac{A_{1}^{2}}{\pi^{2}} \frac{\left(R_{L} / / R_{\text {leak }}\right)^{2}}{R_{L}}
$$

$A_{I}$ in (25) can be expanded using (21) and (24).

$$
P_{\text {del }}=\frac{-\left(4 P_{\text {out }} \sin \frac{\omega t_{d}}{2} R_{s}-V_{\text {in }}\right)}{k_{2}+4 \frac{\left(R_{L} / / R_{\text {leak }}\right)^{2}}{\pi^{4}} \sin ^{2} \frac{\omega t_{d}}{2}} \cdot \frac{\left(R_{L} / / R_{\text {leak }}\right)^{2}}{\pi^{2} R_{L}}
$$

$P_{\text {out }}$ in (26) can then be substituted for using (25) and the resulting expression rearranged to find $P_{d e l}$ in terms of circuit impedances, $R_{s}, k_{2}, R_{L}$ and $R_{\text {leak }}$, the open circuit voltage $v_{i n}$, harvester fundamental frequency $\omega$, and diode on time $t_{d}$.
$P_{\text {del }}=\frac{V_{\text {in }}{ }^{2}}{\left(4 \frac{R_{L}}{\pi^{2}} \sin ^{2} \frac{\omega t_{d}}{2}+\frac{k_{2}\left(R_{L}+R_{\text {leak }}\right)^{2} \pi^{2}}{R_{L} R_{\text {leak }}{ }^{2}}+4 R_{s} \frac{\left(R_{L}+R_{\text {leak }}\right)}{R_{\text {leak }}} \sin \frac{\omega t_{d}}{2}\right)}$

Optimum load resistance for a harvester $R_{L_{-} \text {opt }}$ occurs where maximum power will be delivered to the load. $P_{d e l}$, reaches a maximum when $d P_{d e l} / d R_{L}=0 \cdot d P_{d e l} / d R_{L}$ can be found by differentiating (27) with respect to $R_{L}$ and equating to 0 . Many terms on the right hand side of (27) are independent of $R_{L}$, but the diode on time $t_{d}$ is not, so (27) cannot be differentiated until $t_{d}$ has been defined.

Examination of (9) and (12b) shows that if $m$ is chosen such that $1 / \omega C_{s}$ is closest in magnitude to $m^{2} \omega L_{s}$, then $A_{m} \gg A_{n}$ for all $n \geq 1, m \neq n$. Since observation 1 in Section II $B$ showed that $A_{n}<A_{l}$ for all $n \geq 3$, this means that $1 / \omega C_{s}$ must be closest in value to $\omega L_{s}$ while the higher order coefficients will be much smaller due to the division by the large term $\left(\frac{1}{\omega C_{s}}-n^{2} \omega L_{s}\right)$ in (9). Setting $A_{n} \ll A_{l}$ for all $n \geq 3$ in (5), while also taking (11) into account, gives a simple expression for $i_{i n}$.

$$
i_{\text {in }} \approx A_{1} \cos \omega t
$$

The point where the $i_{\text {in }}$ crosses through zero indicates the diode switching point, $t_{D}$. The zero crossing point in (28) occurs where $t_{D}=T / 4$, so that

$$
\sin \frac{\omega t_{D}}{2}=1 \text {. }
$$

Substituting (29) in (27) and determining $R_{L}$ such that $d P_{d e l} / d R_{L}=0$ gives $R_{L}=R_{L_{-} \text {opt }}$, which achieves maximum delivered output power, $P_{d e l}$ when

$$
R_{L_{-} \text {opt }}=\frac{1}{\sqrt{\frac{4}{k_{2} \pi^{4}}+\frac{1}{R_{\text {leak }}^{2}}+\frac{4 R_{s}}{k_{2} \pi^{2} R_{\text {leak }}}}}
$$

It would now be helpful to also calculate $C_{s_{-} \text {opt }}$, the optimum value for $C_{s}$. $C_{S_{-} \text {opt }}$ can be found in the same manner as $R_{L_{-} \text {opt }}$. Expanding $k_{2}$ in (27) using (22), and again substituting (29) into (27), to find an expression for $P_{d e l}$ in terms of $C_{s}$.

$P_{d e l}=$

$$
\frac{V_{\text {in }}{ }^{2}}{\left.R_{s}{ }^{2}+\left(\frac{1}{\omega C_{s}}-\omega L_{s}\right)^{2}\right]\left(R_{L}+R_{\text {leak }}\right)^{2} \pi^{2}}+4\left(\frac{R_{L}}{\pi^{2}}+R_{s} \frac{\left(R_{L}+R_{\text {leak }}\right)}{R_{\text {leak }}}\right)
$$

This expression for output power delivered to the load resistance can then be differentiated with respect to $C_{s}$. $C_{s_{-} \text {opt }}$ occurs where $d P_{d e l} / d C_{s}=0$ giving

$$
C_{s_{-} \text {opt }}=1 / \omega^{2} L_{s}
$$

The capacitance value given by (32) is the value which, when combined with optimum load resistance given by (30), maximizes harvester output power. Note that $C_{s_{-} \text {opt }}$ given by (32) for a harvester connected to a doubler is the same as the optimum for the harvester connected directly to a load resistor 
without a doubler [1]. Combining (22) and (32) with (30) leads to a simpler expression for $R_{L_{-} \text {opt }}$

$$
R_{L_{-} \text {opt }}=1 / \sqrt{\frac{4}{R_{s}^{2} \pi^{4}}+\frac{1}{R_{\text {leak }}{ }^{2}}+\frac{4}{R_{s} \pi^{2} R_{\text {leak }}}}
$$

If $R_{\text {leak }}$ is several orders of magnitude larger than $R_{s}$ then (33) is further simplified:

$$
R_{L_{-} o p t}=R_{s} \pi^{2} / 2
$$

\section{Regulating doubler output with a dc-dc converter}

Small energy harvesters are commonly used to power wireless sensors using a dc-dc converter for efficient voltage regulation, as shown in Fig. 3. The authors previously published results for an inductive harvester powering a MICAz (MPR2400) 2.4GHz module [20] through a doubler and MAX639 dc-dc converter [1], for which steady-state voltage levels are analyzed below. A start-up circuit [1] is required to monitor the voltage on $C_{\text {stor }}$ and connect the doubler to the dc-dc converter only when sufficient energy is available to supply the wireless sensor. In this configuration, the impedance presented to the doubler is not obvious. If the circuit is to be matched for optimum power transfer, while also ensuring reliable operation, the designer must determine the voltage that $V_{\text {out }}$ will settle to during steady-state operation.

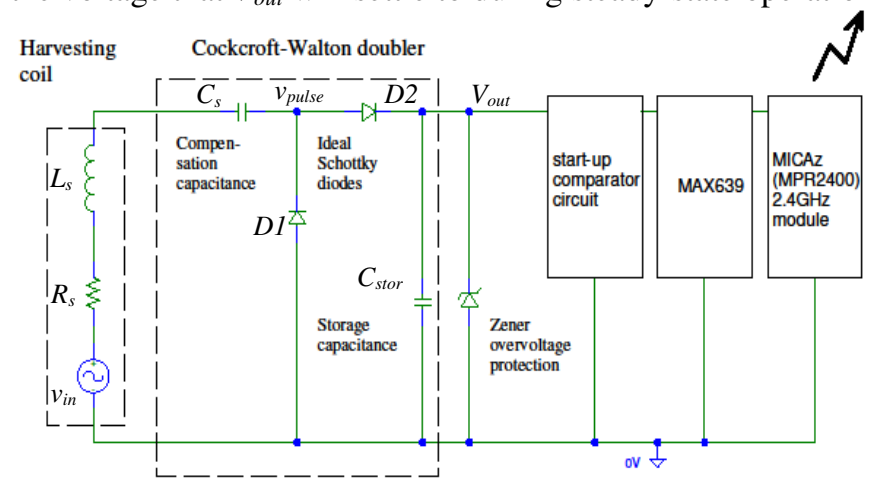

Fig. 3 Inductive energy harvesting powering a wireless sensor through a Cockcroft-Walton doubler, start-up circuit [1] and dc-dc converter.

For this analysis it is assumed that $C_{\text {stor }}$ is large enough to absorb the variation in wireless sensor power dissipation as it switches between quiescent, measurement and transmission states, so that $V_{\text {out }}$ can be considered constant.

The average load impedance $R_{L}$ connected across $V_{\text {out }}$ by the start-up circuit, dc-dc converter and MICAz can be found as a function of $V_{\text {out }}$ and the average output power $P_{\text {sensor }}$ consumed by the combination of wireless sensor, start-up circuit and dc-dc converter.

$$
R_{L}=\frac{V_{\text {out }}^{2}}{P_{\text {sensor }}}
$$

The rms value of the open circuit voltage $V_{\text {in_rms }}$ on an $N$ turn coil wound on a cylindrical core of diameter $D$ and effective relative permeability $\mu_{\text {eff, }}$ ideally linked with magnetic flux density $B$ at frequency $f$ is given by Faraday's Law as [1]:

$$
V_{\text {in_ } r m s}=2 \pi f \mu_{e f f} N \pi(0.5 D)^{2} B
$$

The harvester and doubler can be represented by an ideal dc voltage source in series with the source impedance, as in Fig. 4a. The source impedance has been calculated using (34), since the leakage resistance is not included in the ideal voltage source. $R_{\text {leak }}$ and combined load $R_{L}$ are connected in parallel across the ideal voltage source.

The maximum power available from the harvesting coil is found from $V_{\text {in_rms }}{ }^{2} / 4 R_{s}$. The dc voltage on the ideal voltage source, $V_{\text {source }}$, is such that the power delivered by this ideal source (when matched with $R_{L}=R_{s} \pi^{2} / 2$ and ignoring leakage losses) is equal to the maximum power available from the harvesting coil. Hence

$$
\begin{aligned}
& \frac{V_{\text {source }}{ }^{2}}{4\left(\frac{R_{s} \pi^{2}}{2}\right)}=\frac{V_{\text {in_rms }^{2}}}{4 R_{s}} \\
& V_{\text {source }}=V_{\text {in_rms }} \pi / \sqrt{2}
\end{aligned}
$$

The ideal voltage source and the leakage resistance can then be combined into a Thevenin equivalent voltage source, $V_{t h}$, with Thevenin equivalent source impedance, which, as was discussed in Section II $C$ is equal to $R_{L_{-} o p t}$, as given by (33). Hence

$$
V_{\text {th }}=\frac{V_{\text {source }} R_{\text {leak }}}{R_{\text {leak }}+\frac{R_{s} \pi^{2}}{2}}
$$

The voltage developed across $R_{L}$ is calculated using the voltage divider in Fig. 4b, substituting for $R_{L}$ using (35).

$$
V_{\text {out }}=\frac{\frac{V_{\text {out }}{ }^{2}}{P_{\text {sensor }}}}{\frac{V_{\text {out }}{ }^{2}}{P_{\text {sensor }}}+R_{L_{-} \text {opt }}} V_{\text {th }}
$$

The quadratic in (39) is solved giving

$$
V_{\text {out }}=\frac{V_{\text {th }}}{2} \pm \sqrt{\left(\frac{V_{\text {th }}}{2}\right)^{2}-R_{L_{-} \text {opt }} P_{\text {sensor }}}
$$

This expression gives two possible values for $V_{\text {out }}$, reflecting the fact that either a lower or a higher load impedance than optimum will produce an equivalent power mismatch. The value which the circuit settles to will depend mainly on the value of $V_{\text {out }}$ at start-up.

The action of the start-up circuit is to connect the dc-dc converter input to the doubler output once sufficient energy is stored in $C_{\text {stor }}$. The voltage on $C_{\text {stor }}$ cannot change quickly because of its high capacitance, so the effect of connecting $C_{\text {stor }}$ to the dc-dc converter is to clamp the dc-dc converter input to the voltage on $C_{\text {stor }}$. The available output power from the harvester for any given voltage on $C_{\text {stor }}$ is given by

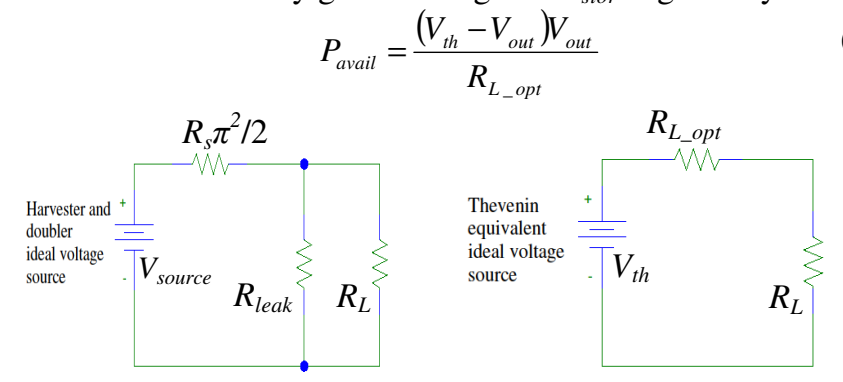

Fig. 4 (a) Harvesting coil and doubler combined into a single ideal dc voltage source, with source impedance given by (34), connected to $R_{\text {leak }}$, and $R_{L}$. (b) Thevenin equivalent of harvester and doubler combined with $R_{\text {leak }}$. 
If $V_{\text {out }}$ at start-up is greater than $V_{t h} / 2$ then $P_{\text {avail }}$ will increase if $V_{\text {out }}$ drops. In contrast, with $V_{\text {out }}$ at start-up smaller than $V_{t h} / 2$ then $P_{\text {avail }}$ will decrease as $V_{\text {out }}$ drops (note that the operation of the doubler places an upper limit on $V_{\text {out }}$ at start-up of $2 V_{\text {in }}$ ). If $P_{\text {avail }}$ is smaller than $P_{\text {sensor }}$ at start-up then $C_{\text {stor }}$ will discharge and $V_{\text {out }}$ will drop. The start-up value for $V_{\text {out }}$ must therefore be chosen to ensure that it is possible to reach the point where $P_{\text {avail }}=P_{\text {sensor }}$ while neither fully discharging $C_{\text {stor }}$ (turning off the start-up circuit) nor over-charging $C_{\text {stor }}$ (turning on the Zener diode).

It is interesting to note, from (41), that maximum harvested power is delivered when $V_{\text {out }}=0.5 V_{t h}$. For a given value of $B$, the designer can therefore optimize coil and electronic circuit design to ensure that $0.5 V_{t h}$ aligns with high dc-dc converter efficiency, and that $P_{\text {avail }}$ when $V_{\text {out }}=0.5 V_{\text {th }}$ is only slightly larger than $P_{\text {sensor }}$. This leads to an economic design in which the harvesting coil is no larger than necessary.

If $C_{\text {stor }}$ is too small to absorb variations in $P_{\text {sensor }}$, then $V_{\text {out }}$ will vary depending on whether the wireless sensor is transmitting, receiving or in sleep-mode. Under these conditions, maximum and minimum $P_{\text {sensor }}$ can be used in (40) to calculate the resulting range for $V_{\text {out }}$.

\section{EXPERIMENTAL SET-UP}

The equations derived in (30), (32) and (34) were verified firstly by simulation and then by the measurements that are presented here. Two quite different coils were measured with a doubler connected to them and output power was measured as a function of $C_{s}$ and $R_{L}$.

\section{A. Coil Design}

Both coils were wound on cylindrical cast iron cores using $0.224 \mathrm{~mm}$ diameter copper wire. In order to explore contrasting coils, a coil with 40,000 turns was measured, as well as one with 8,000 turns. The effective coil resistance at $50 \mathrm{~Hz}$ was an order of magnitude higher than the dc resistance for the coil with 40,000 turns, as documented in [1]. It is important to use the effective coil resistance at $50 \mathrm{~Hz}$ when calculating $R_{L_{-} o p t}$, and not dc resistance. Effective coil resistance at $50 \mathrm{~Hz}$ was measured by compensating for the inductance with a series capacitance and placing the coil in a magnetic field. The effective coil resistance at $50 \mathrm{~Hz}, R_{50 \mathrm{~Hz}}$, is the value which gives the greatest value of $\left(V_{\text {out }}\right)_{r m s}{ }^{2} / R_{50 \mathrm{~Hz}}$. Coil dimensions and impedances are summarized later in Table 1.

\section{B. Cockcroft-Walton doubler design}

The doubler circuit was as shown in Fig. 3. The diodes were very low forward voltage drop Schottky diodes (PMEG2010). $C_{\text {stor }}$ was $100 \mu \mathrm{F}$ aluminum electrolytic for measurements of impedance, and $6800 \mu \mathrm{F}$ for measurements with the wireless sensor. Total $R_{\text {leak }}$ consists of the parallel combination of leakage resistances in the Schottky diodes, storage capacitance and oscilloscope input impedance, and is determined experimentally below.

\section{Generating a Magnetic Field}

Maxwell [21] demonstrated that using three concentric coils it is possible to create an almost uniform magnetic field in the space inside the coils. If $a$ is the radius of the center coil, then the two outer coils must be separated from the center one by a distance $a \sqrt{3 / 7}$ and the radius of each of the outer coils must be $a \sqrt{4 / 7}$. The ampere-turns product of the outer two coils must be equal to 49/64 that of the inner coil. The Maxwell coil set used for these experiments, shown in Fig. 5, had a center coil of radius $760 \mathrm{~mm}$ and outer coils of radius $575 \mathrm{~mm}$. There were 64 turns on the center coil and 49 on each of the outer coils. The current flowing in each coil was equal.

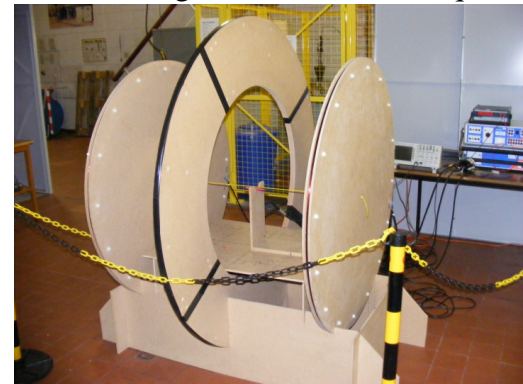

Fig. 5 Maxwell coils used to generate a uniform $B$-field in the laboratory.

\section{MEASUREMENT RESULTS}

Output power delivered by the harvester and doubler to a range of $R_{L}$ values was measured for a selection of $C_{s}$ values using the circuit shown in Fig. 1. Delivered output power was plotted as a function of $R_{L}$ for the 8,000 turn coil in Fig. 6, and for the 40,000 turn coil in Fig. 7. $B$ was set to $18 \mu \mathrm{T}_{r m s}$ and $f$ was $50 \mathrm{~Hz}$ for these measurements. Leakage resistance values for the Schottky and Zener diodes, $C_{\text {stor }}$ and oscilloscope input were accurately determined by applying the appropriate voltage across each leaky component in turn and measuring leakage current. The total leakage resistances were found to be $294 \mathrm{k} \Omega$ with the 40,000 turn coil and $143 \mathrm{k} \Omega$ with the 8,000 turn coil under the voltage conditions present during the experiments. Coil design parameters are summarized in Table 1. Calculated and measured optimum resistance and capacitance are compared in Table 2, while calculated and measured steady-state voltage are compared in Table 3.

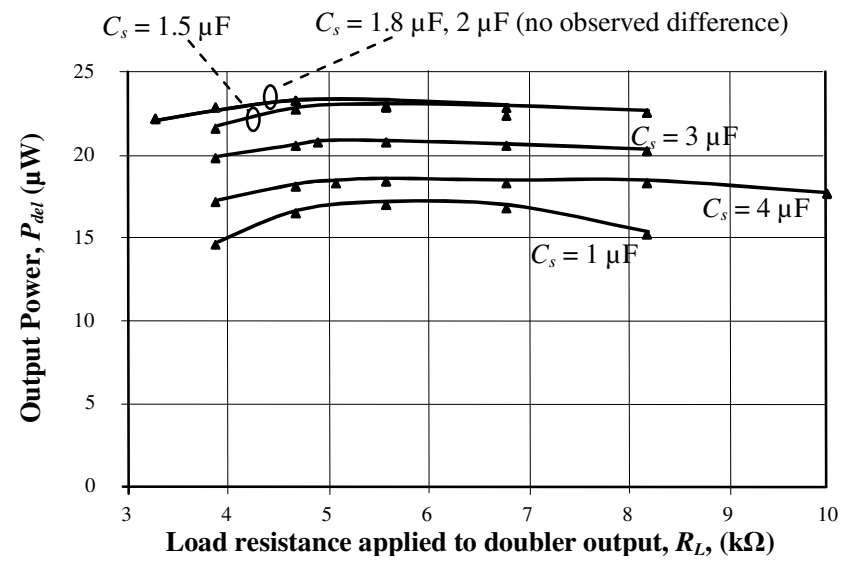

Fig. $6 P_{\text {del }}$ delivered by the 8000-turn coil and doubler, measured on $R_{L}$. Curves are plotted for a range of different values of $C_{s}$. 


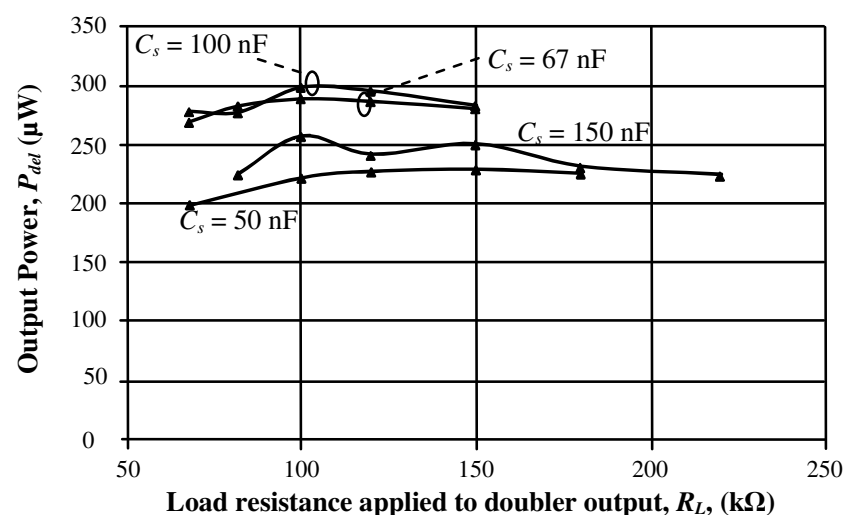

Fig. $7 P_{d e l}$ delivered by the 40,000-turn coil and doubler, measured on $R_{L}$. Curves are plotted for a range of different values of $C_{s}$.

Coil inductance can be estimated from Wheeler's approximations for multi-layered air-cored coils [22] using the depth of the layers of turns $d$, coil length $l$ and the effective relative permeability of the coil $\mu_{e f f}[1]$, giving

$$
L_{s}=\mu_{e f f} \frac{31.6(0.5 D)^{2} N^{2}}{6(0.5 D)+9 l+10 d}
$$

The inductance given by (42) does not allow for the effect of core loss, which modifies the inductance value somewhat, but is close enough for the design of an inductive harvester.

Table 2 compares predicted and measured $R_{L_{-} o p t}$ and $C_{s_{-} o p t}$. These results show good agreement between theory and experiment for calculations of $R_{L_{-} o p t}$. In Table 2, $C_{s_{-} o p t}$ was calculated using calculated $L_{s}$ in (32), which leads to a small discrepancy due to core loss effects.

TABLE I

COIL DESIGN PARAMETERS

\begin{tabular}{|c|c|c|}
\hline & small coil & large coil \\
\hline core length, $l$ & $0.14 \mathrm{~m}$ & $0.5 \mathrm{~m}$ \\
\hline core diameter, $D$ & $0.03 \mathrm{~m}$ & $0.05 \mathrm{~m}$ \\
\hline number of turns, $N$ & 8,000 & 40,000 \\
\hline depth of turn layers, $d$ & $3 \mathrm{~mm}$ & $5 \mathrm{~mm}$ \\
\hline effective relative permeability, $\mu_{\text {eff }}$ & 17 & 22 \\
\hline dc coil resistance, $R_{d c}$ & $598 \Omega$ & $4,100 \Omega$ \\
\hline effective coil resistance $R_{s}($ measured at $50 \mathrm{~Hz})$ & $1 \mathrm{k} \Omega$ & $33 \mathrm{k} \Omega$ \\
\hline calculated coil inductance, $L_{s}$ & $5.9 \mathrm{H}$ & $148 \mathrm{H}$ \\
\hline
\end{tabular}

The harvester and doubler were then connected to a MAX639 dc-dc converter and a MICAz (MPR2400) $2.4 \mathrm{GHz}$ Mote module [20], as shown in Fig. 3. The MAX639 output voltage was set to $3 \mathrm{~V}$ and the MICAz was transmitting every 4 minutes. The power consumption of the MICAz was measured and found to be around $1.3 \mathrm{~mW}$ with supply voltage of $3 \mathrm{~V}$, in contrast to only $0.5 \mathrm{~mW}$ for supply voltage of $2.85 \mathrm{~V}$. MAX639 power consumption was found to be very dependent on $V_{\text {out }}$, but was around $200 \mu \mathrm{W}$ for an input voltage of $3.6 \mathrm{~V}$. $V_{\text {out }}$ was then measured and compared with values predicted using (37), (38) and (40), as shown in Table 3. Measured and calculated $V_{\text {out }}$ are in good agreement for the small coil, but not for the large coil. The large coil has a very high optimum $V_{\text {out }}$ (or very low, if using the lower value), which is incompatible with the electronics. The start-up circuit has connected the harvester at a value of $V_{\text {out }}$ where $P_{\text {avail }}<P_{\text {sensor }}$. $V_{\text {out }}$ settled at $3.25 \mathrm{~V}$ because with $V_{\text {out }}$ at this value, $P_{\text {sensor }}$ has dropped to around $1 \mathrm{~mW}$ which balances it with $P_{\text {avail }}$, which is also around $1 \mathrm{~mW}$ for $V_{\text {out }}=3.25 \mathrm{~V}$.

TABLE 2

COMPARING PREDICTED AND MEASURED INDUCTANCE, OPTIMUM LOAD RESISTANCE AND OPTIMUM SERIES CAPACITANCE

\begin{tabular}{|l|c|c|}
\hline & small coil & large coil \\
\hline measured coil inductance, $L_{s}$ & $4.8 \mathrm{H}$ & $121 \mathrm{H}$ \\
\hline calculated optimum series capacitance, $C_{\text {s_opt }}$ & $1,700 \mathrm{nF}$ & $69 \mathrm{nF}$ \\
\hline measured optimum series capacitance, $C_{\text {s_opt }}$ & $1,900 \mathrm{nF}$ & $100 \mathrm{nF}$ \\
\hline $\begin{array}{l}\text { calculated optimum load resistance, } \\
\text { excluding leakage, } R_{L_{o} \text { opt }}\end{array}$ & $4.9 \mathrm{k} \Omega$ & $162 \mathrm{k} \Omega$ \\
\hline $\begin{array}{l}\text { calculated optimum load resistance, } \\
\text { including leakage, } R_{L_{-o p t}}\end{array}$ & $4.8 \mathrm{k} \Omega$ & $105 \mathrm{k} \Omega$ \\
\hline measured optimum load resistance, $R_{L_{-} o p t}$ & $5 \mathrm{k} \Omega$ & $100 \mathrm{k} \Omega$ \\
\hline efficiency, $R_{L}=R_{L_{-} \text {opt }}$ & $46 \%$ & $63 \%$ \\
\hline efficiency, $R_{L}=10 \mathrm{k} \Omega$ & $39 \%$ & $21 \%$ \\
\hline
\end{tabular}

The effect of impedance match on the doubler output is seen in Table 2. Efficiency of a harvester driving load resistance equal to average wireless sensor input impedance is compared with efficiency of an optimally matched harvester.

The importance of considering the combination of available magnetic field, coil design, electronic design and start-up $V_{\text {out }}$ can be seen by examining results in Table 3. For the small coil $P_{\text {avail }}$ is slightly larger than that delivered, and final $V_{\text {out }}$ is only slightly larger than $0.5 V_{t h}$. This coil is therefore very well matched to the electronic circuit for this value of $B$. The large coil however has the potential to deliver 3 times the power $\left(P_{\text {avail }}=4.4 \mathrm{~mW}\right)$, even with a smaller value of $B$, and is therefore too large for this value of $B$. Moreover, in practice the large coil could not deliver $4.4 \mathrm{~mW}$ because start-up $V_{\text {out }}$ is much less than $0.5 V_{t h}$, although in fact $V_{\text {out }}$ cannot be set close to $0.5 V_{\text {th }}$ because $V_{\text {out }}=0.5 V_{\text {th }}$ would destroy the dc-dc converter, showing that coil and electronics are poorly matched. These formulae therefore allow the designer to quickly assess whether coil and electronic design are well matched to one another and appropriate for the ambient value of $B$.

TABLE 3

COMPARING MEASURED AND CALCULATED DOUBLER OUTPUT VOLTAGE

\begin{tabular}{|l|c|c|}
\hline & small coil & large coil \\
\hline magnetic flux density, $B$ & $80 \mu \mathrm{T}_{r m s}$ & $45 \mu \mathrm{T}_{r m s}$ \\
\hline open circuit coil voltage, $V_{\text {in }}$ & $2.56 \mathrm{~V}_{r m s}$ & $25.5 \mathrm{~V}_{r m s}$ \\
\hline measured coil series resistance, $R_{s}$ & $1 \mathrm{k} \Omega$ & $33 \mathrm{k} \Omega$ \\
\hline total leakage resistance, $R_{\text {leak }}$ & $143 \mathrm{k} \Omega$ & $294 \mathrm{k} \Omega$ \\
\hline $\begin{array}{l}\text { ideal source voltage presented by coil and } \\
\text { doubler, neglecting leakage, } V_{\text {source }}\end{array}$ & $5.7 \mathrm{~V}_{d c}$ & $57 \mathrm{~V}_{d c}$ \\
\hline $\begin{array}{l}\text { Thevenin combined source voltage, } \\
\text { including leakage, } V_{\text {th }}\end{array}$ & $5.5 \mathrm{~V}_{d c}$ & $42 \mathrm{~V}_{d c}$ \\
\hline voltage on doubler output at start-up, $V_{\text {turn_on }}$ & $4.6 \mathrm{~V}_{d c}$ & $5.4 \mathrm{~V}_{d c}$ \\
\hline $\begin{array}{l}\text { power dissipated by start-up circuit, dc-dc } \\
\text { converter and wireless sensor, } P_{\text {sensor }}\end{array}$ & $1.5 \mathrm{~mW}$ & $1.5 \mathrm{~mW}$ \\
\hline $\begin{array}{l}\text { calculated steady state doubler output } \\
\text { voltage, } V_{\text {out }}\end{array}$ & 2 or $3.5 \mathrm{~V}_{d c}$ & 1 or $41 \mathrm{~V}_{d c}$ \\
\hline $\begin{array}{l}\text { measured steady state doubler output voltage } \\
\text { capacitance, } V_{\text {out }}\end{array}$ & $3.6 \mathrm{~V}_{d c}$ & $3.25 \mathrm{~V}_{d c}$ \\
\hline$P_{\text {avail }}, V_{\text {out }}=0.5 V_{t h}$ & $1.6 \mathrm{~mW}$ & $4.4 \mathrm{~mW}$ \\
\hline
\end{tabular}

\section{VALIDATION USING THIRD PARTY HARVESTER RESULTS}

The formula (34) for $R_{L_{-} \text {opt }}$ can be applied to the results published by Bhuiyan et al. [11] for an "enclosing" inductive energy harvester. For a harvesting coil wound using 30 AWG $(0.255 \mathrm{~mm})$ wire the coil series resistance was $9.74 \Omega$ and 
inductance was $327 \mu \mathrm{H}$. Optimum output impedance was measured for this coil driving a single stage Cockcroft-Walton doubler in a $60 \mathrm{~Hz}$ magnetic field. The inductance was not compensated but instead a $220 \mu \mathrm{F}$ capacitor was connected in series. Combining this coil and the series capacitance leads to a source impedance of magnitude $15.6 \Omega$ at $60 \mathrm{~Hz}$. Using (34) and observing that, for such low coil impedance, leakage resistance is several orders of magnitude higher than coil impedance, $R_{L_{-} o p t}$ is predicted to be $78 \Omega$. This agrees very well with the figure of $76 \Omega$ for $R_{L_{-} o p t}$ reported in [11].

\section{CONCLUSIONS}

Inductive harvesting coils terminated with single stage Cockcroft-Walton doublers have been analyzed and measured in order to establish optimum impedance matching conditions. Formulae have been derived which allow series compensation capacitance and load resistance to be optimized and steadystate doubler output voltage to be determined. Two harvesters were built and tested using very different coils, for which experimental results compared well with predicted values. The formula for optimum load resistance was then applied to a harvester presented in the literature by an independent research group. Predicted optimum resistance agreed closely with the independently reported value.

These formulae enable more efficient impedance matching between harvesters and their electronics, and ensure that coil design is compatible with the electronics through knowledge of steady-state voltage. Proper impedance matching and compatibility between harvester and the subsequent circuitry helps to optimize the power available from the energy harvesting device. The analysis presented in this paper will inform the design of energy harvesting devices in future to ensure robust and reliable operation.

While this work has been carried out on harvesters which exhibit strongly inductive source impedances, optimum results were obtained by compensating for the inductance with a resonant capacitor, thereby rendering the source impedance resistive. This method is therefore regarded as being entirely suitable for application to other harvesters, since capacitive source impedances could be absorbed into the doubler, and the resistive part of the source impedance would be matched in the same way as has been presented in this work.

\section{REFERENCES}

[1] N. M. Roscoe and M. D. Judd, "Harvesting Energy from Magnetic Fields to Power Condition Monitoring Sensors", IEEE Sensors Journal, vol. 13, No.6, Jun. 2013, pp. 2263-2270.

[2] M. A. Weimer, T. S. Paing, and R. A. Zane, "Remote area wind energy harvesting for low-power autonomous sensors", Power Electronics Specialists Conference, 2006, pp. 1-5.

[3] Y. K. Tan and S. K. Panda, "Self-Autonomous Wireless Sensor Nodes With Wind Energy Harvesting for Remote Sensing of Wind-Driven Wildfire Spread", IEEE Transactions on Instrumentation and Measurement, Vol. 60, No. 4, April 2011, pp. 1367-1377.

[4] V. Raghunathan; A. Kansal, J. Hsu, J. Friedman, and M. Srivastava, "Design considerations for solar energy harvesting wireless embedded systems", $4^{\text {th }}$ International Symposium on Information Processing in Sensor Networks, 2005, pp. 457-462.

[5] S. Bader and B. Oelmann, "Enabling Battery-Less Wireless Sensor Operation Using Solar Energy Harvesting at Locations with Limited
Solar Radiation", $4^{\text {th }}$ Int. conf. on Sensor Technologies and Applications (SENSORCOMM), July 2010 pp. 602-608,

[6] G.K. Ottman, H.F. Hofmann, A.C. Bhatt and G.A. Lesieutre, "Adaptive piezoelectric energy harvesting circuit for wireless remote power supply", IEEE Transactions on Power Electronics, Vol. 17, Issue: 5 2002 , pp. 669-676.

[7] C.R. Saha, T. O'Donnell, H. Loder, S. Beeby and J. Tudor, "Optimization of an Electromagnetic Energy Harvesting Device", IEEE Trans. on Magnetics, Vol. 42, Issue: 10, 2006 , pp. 3509-3511.

[8] E.P. James, M.J. Tudor, S.P. Beeby, N.R. Harris, P. Glynne-Jones, J.N. Ross and N.M. White, "An investigation of self-powered systems for condition monitoring applications" Sensors and Actuators A, Physical, Vol. 110, Issues 1-3, Feb 2004, pp. 171-176.

[9] A.J. Niskanen, "Magnetic Shape Memory (MSM) Alloys for Energy Harvesting and Sensing Applications," WoWSS 2012, Aalto University 2nd Workshop on Wireless Sensor Systems, Espoo, Finland, Dec 2012.

[10] C. Lu, S.P. Park, V. Raghunathan and K. Roy, "Analysis and design of ultra low power thermoelectric energy harvesting systems", ACM/IEEE International Symposium on Low-Power Electronics and Design (ISLPED), 2010, pp. 183-188.

[11] R.H. Bhuiyan, R.A. Dougal and M. Ali, "A Miniature Energy Harvesting Device for Wireless Sensors in Electric Power System", IEEE Sensors Journal, Vol. 10, Issue: 7, 2010 , pp. 1249-1258.

[12] T. Taithongchai and E. Leelarasmee, "Adaptive electromagnetic energy harvesting circuit for wireless sensor application", 6th International Conference on Computer, Telecommunications and Information Technology, Electrical Engineering/Electronics, 2009, Vol. 1, pp. 278281.

[13] R. Moghe, Y. Yang, F. Lambert and D. Divan, "Design of a Low Cost Self-Powered Stick-on Current and Temperature Wireless Sensor for Utility Assets," IEEE Energy Conversion Congress and Exposition (ECCE), 12-16 Sept. 2010, pp. $4453-4460$

[14] R. Moghe, D. Divan and F. Lambert, "Powering low-cost utility sensors using energy harvesting", Proceedings of the 14th European Conference on Power Electronics and Applications (EPE 2011), 2011, pp. 1-10.

[15] M. Zhu, M.D. Judd and P.J. Moore, "Energy Harvesting in Substations for Powering Autonomous Sensors", Third International Conference on Sensor Technologies and Applications, 2009, pp. 246-251.

[16] H. Zangl, T. Bretterklieber and G. Brasseur, "Energy Harvesting for Online Condition Monitoring of High Voltage Overhead Power Lines", Instrumentation and Measurement Technology Conference Proceedings, 2008, pp. 1364-1369.

[17] S. Cheng, Y. Jin, Y. Rao and D. P. Arnold, "An Active Voltage Doubling AC/DC Converter for Low-Voltage Energy Harvesting Applications," IEEE Trans. Power electronics, vol. 26, No.8, Aug. 2011, pp. 2256-2265.

[18] S. Qi, R. Shuttleworth, S. Olutunde Oyadiji and J. Wright, "Design of a Multi-Resonant Beam for Broadband Piezo-Electric Harvesting", Smart Materials and Structures 19, 2010

[19] A. Rahimi, O. Zorlu, A. Muhtaroglu and H. Kulah, "Fully Self-Powered Electromagnetic Energy Harvesting System with Highly Efficient Dual Rail Output”, IEEE Sensors Journal, Vol. 12, No. 6, June 2012.

[20] MICAz wireless sensor, (MPR2400) $2.4 \mathrm{GHz}$ Mote, http:// www.openautomation.net/uploadsproductos/micaz_datasheet.pdf, accessed Jul 2013.

[21] Clerk-Maxwell, James (1873). Treatise on Electricity and Magnetism. Oxford: The Clarendon Press, Page 319.

[22] H.A. Wheeler, "Simple Inductance Formulas for Radio Coils", Proc. I.R.E., Vol 16, Oct.1928, p.1398.

Nina M. Roscoe (née Tilston) is a Research Fellow at the University of Strathclyde where she has been researching energy harvesting for condition monitoring sensors. Nina spent 5 years designing audio circuitry for Linn Products, and 8 years designing Radar Receivers at GEC-Marconi. Nina received her $\mathrm{PhD}$ in high-linearity $\mathrm{RF}$ amplifiers at Heriot-Watt University in 2005.

Martin D. Judd (M'2002-SM'2004) is a Professor at the University of Strathclyde, whose fields of interest include high frequency electromagnetics, generation and measurement of fast transients, partial discharges and energy harvesting. Martin spent 4 years working for Marconi Electronic Devices Ltd followed by 4 years with EEV Ltd. He received his $\mathrm{PhD}$ from the University of Strathclyde in 1996 for research into the excitation of UHF signals by partial discharges in gas insulated switchgear. 